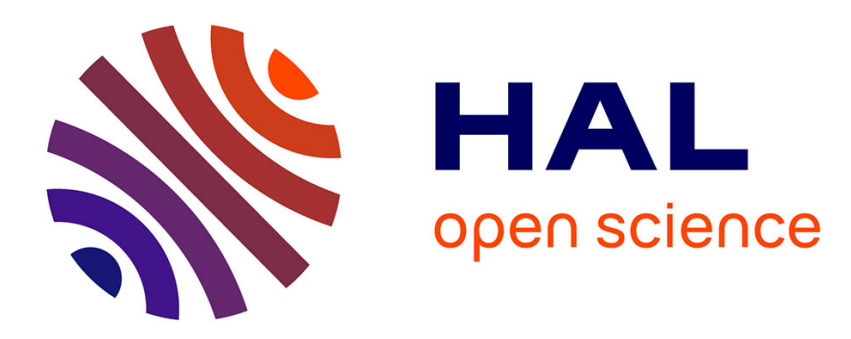

\title{
Initial data identification for the one-dimensional Burgers equation
}

Thibault Liard, Enrique Zuazua

\section{To cite this version:}

Thibault Liard, Enrique Zuazua. Initial data identification for the one-dimensional Burgers equation. 2020. hal-03028457v2

\section{HAL Id: hal-03028457 \\ https://hal.science/hal-03028457v2}

Preprint submitted on 16 Dec 2020

HAL is a multi-disciplinary open access archive for the deposit and dissemination of scientific research documents, whether they are published or not. The documents may come from teaching and research institutions in France or abroad, or from public or private research centers.
L'archive ouverte pluridisciplinaire HAL, est destinée au dépôt et à la diffusion de documents scientifiques de niveau recherche, publiés ou non, émanant des établissements d'enseignement et de recherche français ou étrangers, des laboratoires publics ou privés. 


\title{
Initial data identification for the one-dimensional Burgers equation
}

\author{
Thibault Liard ${ }^{1}$ and Enrique Zuazua ${ }^{2}$
}

\begin{abstract}
In this paper, we study the problem of identification for the one-dimensional Burgers equation. This problem consists in identifying the set of initial data evolving to a given target at a final time. Due to the property of non-backward uniqueness of Burgers equation, there may exist multiple initial data leading to the same given target. In [12], [16], the authors fully characterize the set of initial data leading to a given target using the classical Lax-Hopf formula. In this note, an alternative proof based only on generalized backward characteristics is given. This leads to the hope of investigate systems of conservation laws in one dimension where the classical Lax-Hopf formula doesn't hold anymore. Moreover, numerical illustrations are presented using as a target, a function optimized for minimum pressure rise in the context of sonic-boom minimization problems. All of initial data leading to this given target are constructed using a wave-front tracking algorithm.
\end{abstract}

\section{INTRODUCTION}

\section{A. Sonic-boom minimization}

A supersonic airplane, flying above the speed of sound, creates a near-field pressure disturbance. This near-field pressure propagates via an augmented Burgers equation [11], [26] as follows

$$
\left\{\begin{array}{c}
\frac{\partial P}{\partial \sigma}=P \frac{\partial P}{\partial \tau}+\frac{1}{\Gamma} \frac{\partial^{2} P}{\partial^{2} \sigma}+\sum_{\nu} \frac{C_{\nu}}{\theta_{\nu}} \int_{-\infty}^{\tau} e^{\frac{(\xi-\tau)}{\theta_{\nu}}} \frac{\partial^{2} P(\xi)}{\partial \tau^{2}} d \xi \\
\quad-\frac{1}{2 G} \frac{\partial G}{\partial \sigma} P+\frac{1}{2 \rho_{0} c_{0}} \frac{\partial\left(\rho_{0} c_{0}\right)}{\partial \sigma} P \\
P\left(\sigma_{0}, \cdot\right)=P_{0}(\cdot) .
\end{array}\right.
$$

Above, $P=P(\sigma, \tau)$ is the pressure disturbance, $\sigma$ is the distance from the airplane, $\tau$ is the time and the nearfield pressure is localized at $\sigma_{0}$. For a description of other parameters, we refer to [11], [26], [2]. When the pressure disturbance reaches the ground level, it creates two subsequent loud bangs with a short time lapse in between, so-called sonicboom [3], see Figure 1.

The sonic-boom effects could be minimized by tailoring the shape of the airplane [24]. The near-field pressure is approximated using F-functions theory [27] from the shape of the airplane. We confine our study to the propagation of the sonic-boom from the near-field of the plane down to a desired ground target. More precisely, given a desired ground target $P^{T}$ and the distance of the propagation $\Sigma$, our aim

1 Thibault Liard is with LS2N, Ecole Centrale de Nantes \& CNRS UMR 6004, F-44000 Nantes, France., Thibault. Liarde ls 2n. fr.

2 Enrique Zuazua is with Chair in Applied Analysis, Alexander von Humboldt-Professorship, Department of Mathematics FriedrichAlexander-Universitat, Erlangen-Nurnberg, 91058 Erlangen, Germany, Departamento de Matemáticas, Universidad Autónoma de Madrid, 28049 Madrid, Spain and Chair of Computational Mathematics, Fundación Deusto Av. de las Universidades 24, 48007 Bilbao, Basque Country, Spain, enrique.zuazua@fau.de.

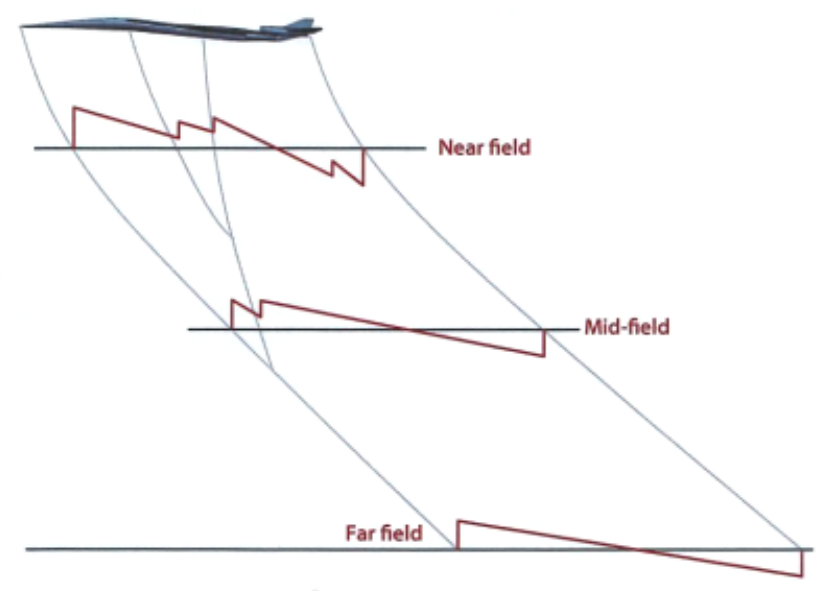

Fig. 1: Evolution of pressure disturbances. The near-field disturbance propagates away from the aircraft to the far-field generating a classic $\mathrm{N}$-wave consisting only of a leading and trailing shock. Extract from [3].

is to identify the set of near-field pressure disturbances $P_{0}$ leading to $P^{T}$ at $\sigma=\Sigma$ along augmented Burgers evolution at the ground level, i.e $P(\Sigma, \cdot)=P^{T}$ where $P$ solution of (1) with initial data $P_{0}$. We refer the reader to [3] for a detailed review on the state of the art related to sonic-boom minimization problem.

In this paper, we take into account the non linear hyperbolic dynamic of (1) disregarding the non-local term and the diffusion effects. Hence, the augmented Burgers equation is reduced to a one-dimensional Burgers equation (2) using the change of variable $u(t, x)=P(t,-x)$. In Section III we construct multiple approximate initial data $P_{0}$ leading to ground-boom signature $P^{T}$ optimized for minimum pressure rise, see [3, Figure 2].

\section{B. Presentation of the problem}

We consider the one-dimensional scalar conservation laws

$$
\left\{\begin{array}{l}
\partial_{t} u(t, x)+\partial_{x} f(u(t, x))=0, \quad(t, x) \in \mathbb{R}^{+} \times \mathbb{R}, \\
u(0, x)=u_{0}(x),
\end{array}\right.
$$

where $u$ is the state, $u_{0}$ is the initial state and the flux function $f$ is defined by $f(u)=\frac{u^{2}}{2}$. Kruzkov's theory [20] provides existence and uniqueness of a solution of (2) with bounded initial datum $u_{0} \in L^{\infty}(\mathbb{R})$. 
Let $T>0$ a final time and $u^{T}$ a target function. As 21 is time-irreversibility, some conditions on $u^{T}$ need to be imposed for it to be attainable. This is shown in [12, Theorem 3.1, Corollary 3.2], [19, Corollary 1] or [17] where they prove that $u^{T}$ is truly attainable in an exact manner by a solution of (2) if and only if $u^{T}$ satisfies the one-sided Lipschitz condition [6], [18], [23], [15], i.e

$$
\partial_{x} u^{T} \leq \frac{1}{T} \text { in } \mathcal{D}^{\prime}(\mathbb{R})
$$

Due to the property of non-backward uniqueness of (2), there may exist multiple initial data leading to the same attainable target $u^{T}$, as seen in Figure 3 In [19], the authors prove that the set of initial data evolving to an attainable target $u^{T}$ is a convex set. Later on, the aforementioned set was fully characterized in [12], [16] using the classical Lax-Hopf formula [21, Theorem 2.1].

In [9], [10], [1], [9], initial data identification of Burgers equation is regarded as an optimal control problem

$$
\min _{u_{0}} J_{0}\left(u_{0}\right):=\left\|u^{T}(\cdot)-u(T, \cdot)\right\|_{L^{2}(\mathbb{R})}
$$

To solve the optimal control problem (4), some difficulties arise from a theoretical and numerical point of view. Because of the presence of discontinuities (called shocks) in the solution $u$ of (2), the derivative of the cost function $J_{0}$ in (4) is regarded in a weak sense by requiring strong conditions on the set of initial data [7], [8], [4], [5]. This leads to require that entropy solutions of (2) have a finite number of non-interacting jumps. When $J_{0}$ is weakly differentiable, gradient descent methods have been implemented in [9], [10], [1] to solve numerically the optimal problem (4). In the cases where it was applied successfully, only one possible initial datum emerges, namely the backward entropy solution, see Section $\mathrm{I}-\mathrm{C}$. To find some multiple minimizers, the authors in [19] use a filtering step in the backward adjoint solution.

In our paper, an alternative proof of the characterization of the set of initial data in [12], [16] is given using only backward generalized characteristics. This leads to the hope of investigate systems of conservation laws in one dimension. More precisely, [12, Theorem 4.1] cannot be extend to systems as Euler equations, Saint-Venant equations or Aw-RascleZhang model since Lax-Hopf formula does not hold anymore. Moreover, a wave-front tracking method is implemented to construct numerically all of them. In the simulations, the target function is optimized for minimum pressure rise in the context of sonic-boom minimization problems.

\section{The backward-forward method}

For a sake of completeness, we recall the definition of a weak-entropy solution of (2).
Definition 1. - We say that $u \in L^{\infty}\left(\mathbb{R}^{+} \times \mathbb{R}\right) \cap$ $C^{0}\left(\mathbb{R}^{+}, L_{\text {loc }}^{1}(\mathbb{R})\right)$ is a weak solution if for all $\varphi \in$ $C_{c}^{1}\left(\mathbb{R}^{2}, \mathbb{R}\right)$,

$\int_{\mathbb{R}^{+}} \int_{\mathbb{R}}\left(u \partial_{t} \varphi+f(u) \partial_{x} \varphi\right) d x d t+\int_{\mathbb{R}} u_{0}(x) \varphi(0, x) d x=0$.

- We say that $u \in L^{\infty}\left(\mathbb{R}^{+} \times \mathbb{R}\right) \cap C^{0}\left(\mathbb{R}^{+}, L_{\text {loc }}^{1}(\mathbb{R})\right)$ is a weak-entropy solution if $u$ is a weak solution and for every $k \in \mathbb{R}$, for all $\varphi \in C_{c}^{1}\left(\mathbb{R}^{2}, \mathbb{R}^{+}\right)$,

$$
\begin{array}{r}
\int_{\mathbb{R}^{+}} \int_{\mathbb{R}}\left(|u-k| \partial_{t} \varphi+\operatorname{sgn}(u-k)(f(\rho)-f(k)) \partial_{x} \varphi\right) d x d t \\
+\int_{\mathbb{R}}\left|u_{0}-k\right| \varphi(0, x) d x \geq 0 .
\end{array}
$$

Kruzkov's theory [20] provides existence and uniqueness of a weak-entropy solution $(t, x) \rightarrow S_{t}^{+}\left(u_{0}\right)(x)$ of (2) with initial datum $u_{0} \in L^{\infty}(\mathbb{R})$. For a given function $u^{T}$, we introduce the backward function $(t, x) \rightarrow S_{t}^{-}\left(u^{T}\right)(x)$ as follows: for every $t \in[0, T]$, for a.e $x \in \mathbb{R}$,

$$
S_{t}^{-}\left(u^{T}\right)(x)=S_{t}^{+}\left(x \rightarrow u^{T}(-x)\right)(-x) .
$$

Remark 1. The solutions $S_{t}^{+}\left(u_{0}\right)$ and $S_{t}^{-}\left(u^{T}\right)$ may be regarded as the zero viscosity limit of the solutions $S_{t}^{+, \varepsilon}\left(u_{0}\right)$ and $S_{T}^{-, \varepsilon}\left(u^{T}\right)$ respectively where $S_{t}^{+, \varepsilon}\left(u_{0}\right)$ and $S_{t}^{-, \varepsilon}\left(u^{T}\right)$ are defined as follows: $S_{t}^{+, \varepsilon}\left(u_{0}\right)$ is the solution of the following viscous Burgers equation

$\begin{cases}\partial_{t} u(t, x)+\partial_{x} f(u(t, x))=\varepsilon \partial_{x x}^{2} u(t, x), & (t, x) \in \mathbb{R}^{+} \times \mathbb{R}, \\ u(0, \cdot)=u_{0}(x), & x \in \mathbb{R},\end{cases}$ and $S_{t}^{-, \varepsilon}\left(u_{0}\right)$ is the solution of the following backward equation

$\begin{cases}\partial_{t} u(t, x)+\partial_{x} f(u(t, x))=-\varepsilon \partial_{x x}^{2} u(t, x), & (t, x) \in \mathbb{R}^{+} \times \mathbb{R}, \\ u(T, \cdot)=u^{T}(x), & x \in \mathbb{R} .\end{cases}$

Using the change of variable $(t, x) \rightarrow(T-t,-x)$, we notice that the backward equation above is well-defined. Thus, $S_{T}^{-}\left(u^{T}\right)$ is called the backward entropy solution.

The backward-forward method consists in solving backward in time the PDE 2) with final target $u^{T}$ and then solving it forward in time with initial datum $S_{T}^{-}\left(u^{T}\right)$, the solution of the backward PDE.

For any attainable target $u^{T}$, we have $S_{T}^{+}\left(S_{T}^{-}\left(u^{T}\right)\right)=u^{T}$ as seen in [12, Theorem 3.1, Corollary 3.2] and [19, Corollary $1]$.

\section{MAIN RESULTS}

Fix $u^{T} \in L^{\infty}(\mathbb{R})$, we introduce the set

$$
\mathcal{I}\left(u^{T}\right)=\left\{u_{0} \in L^{\infty}(\mathbb{R} ; \mathbb{R}): S_{T}^{+}\left(u_{0}\right)=u^{T}\right\} .
$$

From [12, Corollary 3.2], $\mathcal{I}\left(u^{T}\right) \neq \emptyset$ if and only if a suitable representative of $u^{T}$ satisfies the Oleinik condition [6], [18], [23], [15], i.e for every $x \in \mathbb{R}$ and $y \in \mathbb{R}^{+} \backslash\{0\}$,

$$
f^{\prime}\left(u^{T}(x+y)-f^{\prime}\left(u^{T}(x)\right)\right) \leq \frac{y}{T} .
$$


When $f \in B V_{l o c}(\mathbb{R})$, the limits $\lim _{x \rightarrow x_{0}, x>x_{0}} f(x):=f\left(x_{0}+\right)$ and $\lim _{x \rightarrow x_{0}, x<x_{0}} f(x):=f\left(x_{0}-\right)$ exist.

Theorem 1. Let $T>0$ and a suitable representation of $u^{T} \in$ $L^{\infty}(\mathbb{R})$ satisfies the Oleinik condition (7). Then the initial data $u_{0} \in L^{\infty}(\mathbb{R})$ verifies $S_{T}^{+}\left(u_{0}\right)=u^{T}$ if and only if the following statements holds. For any $(x, y) \in X\left(u^{T}\right) \times \mathbb{R}$

$$
\int_{x-T f^{\prime}\left(u^{T}(x)\right)}^{y} S_{T}^{-}\left(u^{T}\right)(s) d s \leq \int_{x-T f^{\prime}\left(u^{T}(x)\right)}^{y} u_{0}(s) d s,
$$

For any $(x, y) \in X\left(u^{T}\right)^{2}$,

$$
\int_{x-T f^{\prime}\left(u^{T}(x)\right)}^{y-T f^{\prime}\left(u^{T}(y)\right)} S_{T}^{-}\left(u^{T}\right)(s) d s=\int_{x-T f^{\prime}\left(u^{T}(x)\right)}^{y-T f^{\prime}\left(u^{T}(y)\right)} u_{0}(s) d s,
$$

where $\left.X\left(u^{T}\right)=\left\{x \in \mathbb{R}, u^{T}(x-)=u^{T}(x+)\right\}\right)$ and $S_{T}^{-}\left(u^{T}\right)$ is defined in (5).

Remark 2. When $u^{T} \in L^{\infty}(\mathbb{R})$ satisfies the Oleinik condition (7), then $u^{T} \in B V_{l o c}(\mathbb{R})$. Thus, $X\left(u^{T}\right)$ is well-defined.

\section{Simulations}

Let $u^{T}$ a given target defined by

$$
u^{T}(x)=\min \left(1, \max \left(-1, \frac{1}{2}(x-1)-1\right)\right) \mathbb{1}_{(0,6)}(x) .
$$

Note that $P^{T}: x \rightarrow u^{T}(-x)$ is of the form of the groundboom signature optimized for minimum pressure rise, see [3] Figure 2].

To solve (2) with initial datum $u_{0} \in B V(\mathbb{R})$, we have implemented a wave-front tracking algorithm, proposed by Dafermos [14], in the software Matlab. If $\underline{u} \leq u_{0} \leq \bar{u}$, we introduce the state mesh $\mathcal{M}_{n}:=\underline{u}+(\bar{u}-\underline{u})\left(2^{-n} \mathbb{N} \cap[0,1]\right)$ and the approximate wave-front tracking solution of $S_{t}^{+}\left(u_{0}\right)$ is denoted by $S_{t}^{+, n}\left(u_{0}\right): \mathbb{R} \rightarrow \mathcal{M}_{n}$ with $n \in \mathbb{N}$ the discretization parameter.

Step 1. We construct an approximate backward entropy solution

$$
S_{t}^{-, n}\left(u^{T}\right): x \rightarrow S_{t}^{+, n}\left(x \rightarrow u^{T}(-x)\right)(-x),
$$

with $\underline{u}=-1, \bar{u}=1$ and $n=6$. An illustration of $S_{t}^{-, n}\left(u^{T}\right)(x)$ in the plane $(x, t)$ is given in Figure $2 \mathrm{a}$ The solution $S_{t}^{-, n}\left(u^{T}\right)$ at time $t=T$ is plotted with respect to $x$ in Figure $2 \mathrm{~b}$. In figure $2 \mathrm{c}$, the approximate solution $(t, x) \rightarrow S_{t}^{+, n}\left(S_{T}^{-, n}\left(u^{T}\right)\right)(x)$ of (2) with initial data $S_{T}^{-, n}\left(u^{T}\right)$ is plotted in the plane $(x, t)$. Note that $S_{t}^{+, n}\left(S_{T}^{-, n}\left(u^{T}\right)\right)$ at time $t=T$ is an approximate function of $u^{T}$, see Figure 2d

Step 2. From (8) and (9), we construct the set of initial data $u_{0}$ leading to $u^{T}$ along forward entropy evolution of Burgers equation (2). In Figure 3, four of them are plotted in 3. As a consequence, $S_{T}^{+, n}\left(u_{0}\right)$ is an approximate function of $u^{T}$. Note that, from $(9)$, for any $u_{0}$ satisfying $S_{T}^{+}\left(u_{0}\right)=u^{T}$, for any $x \in(-\infty, 0) \cup(2,4) \cup(6,+\infty), u_{0}(x)=S_{T}^{-}\left(u^{T}\right)(x)$,

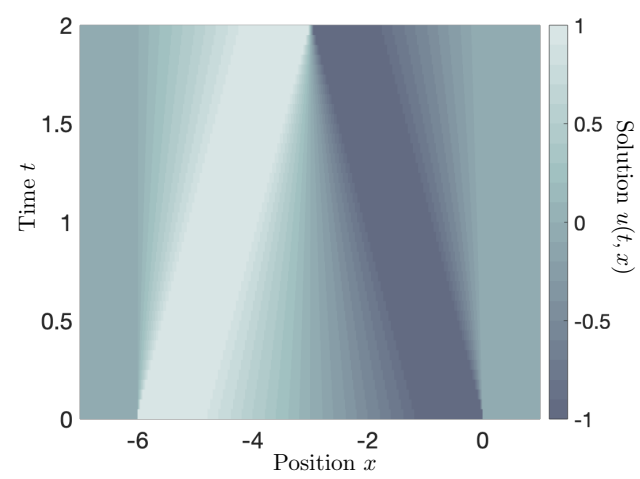

(a) $(x, t) \rightarrow S_{t}^{-, n}\left(u^{T}\right)(-x)$

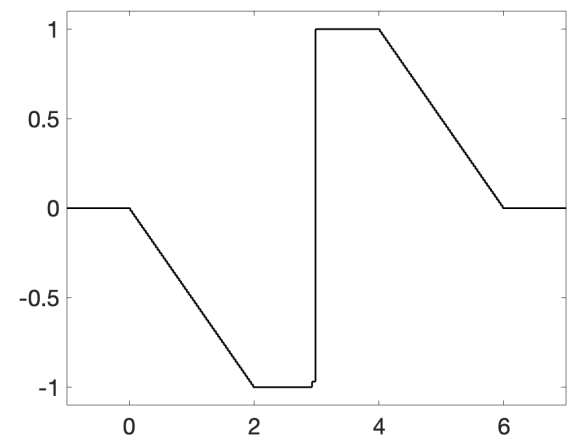

(b) $x \rightarrow S_{T}^{-, n}\left(u^{T}\right)(x)$

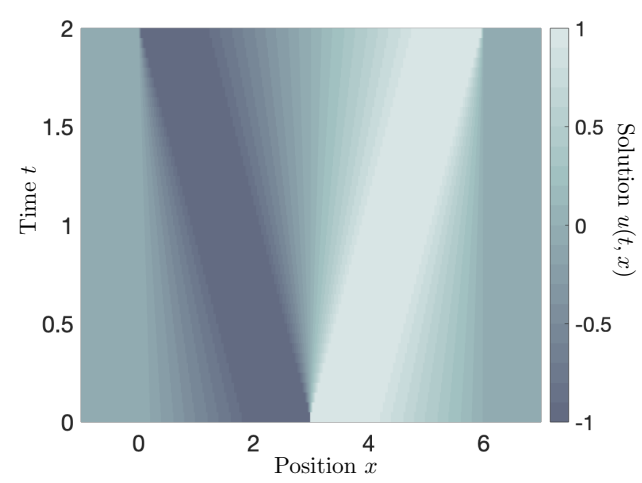

(c) $(x, t) \rightarrow S_{t}^{+, n}\left(S_{T}^{-, n}\left(u^{T}\right)\right)(x)$

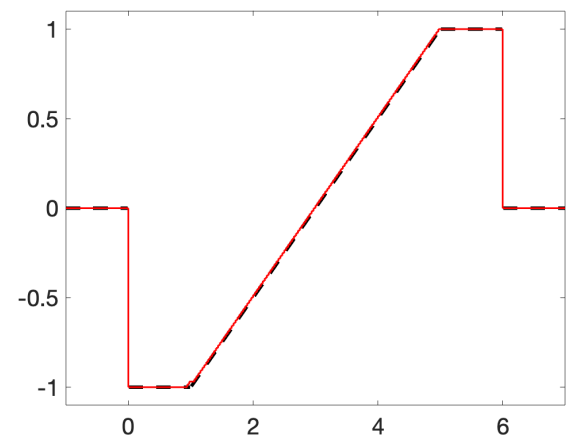

(d) $u^{T}(--)$ and $x \rightarrow S_{T}^{+, n}\left(S_{T}^{-, n}\left(u^{T}\right)\right)(x)(-)$

Fig. 2: $T=2, n=6$. Construction of an approximate backward entropy solution of $S_{t}^{-}\left(u^{T}\right)$ and an approximate solution of $S_{T}^{+}\left(S_{T}^{-}\left(u^{T}\right)\right)$ of $u^{T}$ defined in (10) 
see Figure $2 \mathrm{~b}$ and Figure 3 .

Back to the sonic-boom minimization problem, using the change of variable $P(t, x)=u(t,-x)$, we construct theoretically and numerically the set of near-pressure disturbances $P_{0}$ leading to ground bound signature $P^{T}$ optimized for minimum pressure rise. Among this set, a selection of admissible $P_{0}$ could be imposed to ensure feasible aircraft design (for instance aerodynamic lift) [3, Section 3.3], [22], [25].

\section{Proof of Theorem 1}

The proof of Theorem 1 is based on the two following lemma and the notion of generalized backward characteristics. This latter notion is an important tool for the study of analytical and geometric properties of weak solutions of (2). We refer to [15, Section 10, Section 11] for more details.

Lemma 1. For every $\left(\gamma_{1}, \gamma_{2}\right) \in C^{0,1}([0, T] ; \mathbb{R})^{2}$, for every $t_{1}<t_{2}$,

$$
\begin{aligned}
\int_{\gamma_{1}\left(t_{1}\right)}^{\gamma_{2}\left(t_{1}\right)} u\left(t_{1}, x\right) d x+\int_{t_{1}}^{t_{2}} F\left(u\left(t, \gamma_{1}(t)-\right)\right) d t \\
\quad=\int_{\gamma_{1}\left(t_{2}\right)}^{\gamma_{2}\left(t_{2}\right)} u\left(t_{2}, x\right) d x+\int_{t_{1}}^{t_{2}} F\left(u\left(t, \gamma_{2}(t)+\right)\right) d t
\end{aligned}
$$

with $u$ a weak-entropy solution of (2) and for every $t \in \mathbb{R}^{+}$, for every $\gamma \in C^{0,1}([0, T] ; \mathbb{R})$,

$$
F(u(t, \gamma(t) \pm))=f(u(t, \gamma(t) \pm)-\dot{\gamma}(t) u(t, \gamma(t) \pm) .
$$

The proof of Lemma 1 is given in [13, Lemma 3.2]. We introduce the function $Q: \mathbb{R}^{3} \rightarrow \mathbb{R}$ defined by

$Q(u, v, w)= \begin{cases}f(v)-f(u)-\frac{f(u)-f(w)}{u-w}(v-u), & \text { if } u \neq w, \\ f(v)-f(u)-f^{\prime}(u)(v-u), & \text { if } u=w .\end{cases}$

Since $f$ is strictly convex, we deduce the following Lemma

Lemma 2. Let $u, w \in \mathbb{R}$.

- If $v \in[u, w]$ with $u \leq w, Q(u, v, w) \leq 0$.

- If $v \notin[u, w], Q(u, v, w) \geq 0$.

- If $u=v, Q(u, v, w)=0$.

Proof of Theorem 1. Let $u_{0} \in L^{\infty}(\mathbb{R})$ and assuming that a suitable representation of $u^{T} \in L^{\infty}(\mathbb{R})$ satisfies the Oleinik condition (7). We denote by $u$ the weak-entropy solution of (2) with initial data $u_{0}$ and $u^{*}$ the weak-entropy solution of (2) with initial data $u_{0}^{*}:=S_{T}^{-}\left(u^{T}\right)$ where $S_{T}^{-}\left(u^{T}\right)$ is defined in (5).

Assuming that $u(T, \cdot)=u^{T}(\cdot)$. Let $(x, y) \in X\left(u^{T}\right) \times \mathbb{R}$ with $x-T f^{\prime}\left(u^{T}(x)\right)<y$. Since $u^{T} \in L^{\infty}(\mathbb{R})$ satisfies the one-sided Lipschitz condition, from [12, Theorem 3.1], we have $u^{*}(T, \cdot)=u^{T}(\cdot)$. From Lemma 1 with $t_{1}=0$ and $t_{2}=T$ applied with $u_{0}$ and $u_{0}^{*}$ and using that $u(T, \cdot)=u^{*}(T, \cdot)=$ $u^{T}$, we have

$$
\begin{aligned}
\int_{\gamma_{1}(0)}^{\gamma_{2}(0)}\left(u_{0}(s)-\right. & \left.u_{0}^{*}(s)\right) d s= \\
& \int_{0}^{T} F\left(u\left(t, \gamma_{2}(t)+\right)\right)-F\left(u^{*}\left(t, \gamma_{2}(t)+\right)\right) d t \\
- & \int_{0}^{T}\left(F\left(u\left(t, \gamma_{1}(t)-\right)\right)-F\left(u^{*}\left(t, \gamma_{1}(t)-\right)\right)\right) d t
\end{aligned}
$$
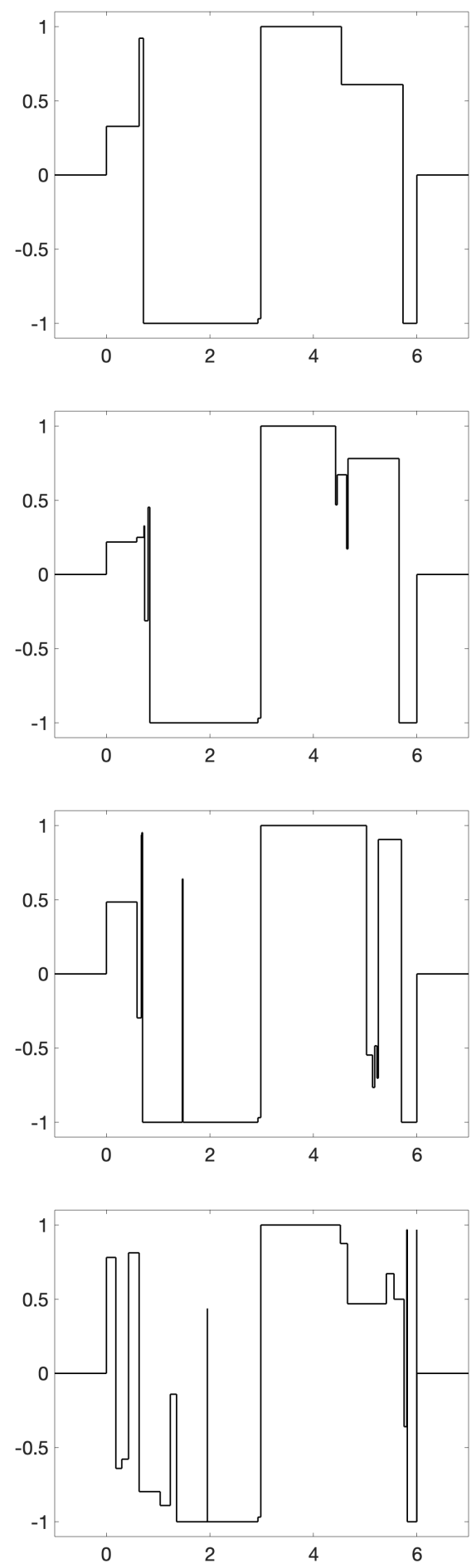

Fig. 3: $T=2, n=6$ and $u^{T}$ defined in 10). Plotting of four different $u_{0}$ with respect to $x$ yielding solutions of Burgers equation that coincide with $u^{T}$ at time $t=T$. 
for every $\left(\gamma_{1}, \gamma_{2}\right) \in C^{0,1}([0, T] ; \mathbb{R})$.

- Since $x \in X\left(u^{T}\right)$, we have $u^{T}(x-)=u^{T}(x+)=$ $u(T, x-)=u(T, x+)$. Thus, from [15, Theorem 11.1.3], the minimal backward generalized characteristic $\xi_{-}$and the maximal backward generalized characteristic $\xi_{+}$, associated to $u$ (or $u^{T}$ or $u^{*}$ ), emanating from $(T, x)$ coincide over $[0, T]$, i.e $\xi_{-}(t)=\xi_{+}(t)$ for every $t \in[0, T]$. Moreover, $\xi_{-}$is a straight line with slope $f^{\prime}\left(u^{T}(x-)\right)=$ $f^{\prime}\left(u^{T}(x+)\right)$ and $\xi_{-}(T)=x-T f^{\prime}\left(u^{T}(x)\right)$. The function $\xi:[0, T] \rightarrow \mathbb{R}$ defined by $\xi(t)=\xi_{-}(T-t)$ is a forward characteristic associated with $u$ (or $u^{T}$ or $u^{*}$ ), issuing from $\left(0, x-T f^{\prime}\left(u^{T}(x)\right)\right.$.

- There exists a forward generalized characteristic $\xi^{*} \in$ $C^{0,1}([0, T] ; \mathbb{R})$, associated with $u^{*}$, issuing from $(0, y) \sqrt{1}$ From [12, Lemma 7.2], $(t, x) \rightarrow S_{t}^{-}\left(u^{T}\right)(x)$ is Lipschitz continuous on any compact $(0, T) \times \mathbb{R}$, thus $\xi^{*}$ is a straight line with slope $f^{\prime}\left(u^{*}\left(\frac{T}{2}, \xi^{*}\left(\frac{T}{2}\right)\right)\right)$. Thus, for every $s \in$ $(0, T), u^{*}\left(s, \xi^{*}(s)-\right)=u^{*}\left(s, \xi^{*}(s)+\right)$.

From [15, Corollary 11.1.2], since $\xi$ and $\xi^{*}$ are distinct generalized characteristics for (2) associated with the admissible weak solution $u^{*}$, which are shock-free on the time interval $[0, T]$. Then $\xi$ and $\xi^{*}$ cannot intersect for any $t \in(0, T)$ as seen in Figure 4 From [15, Theorem 10.2.3] and (12) with

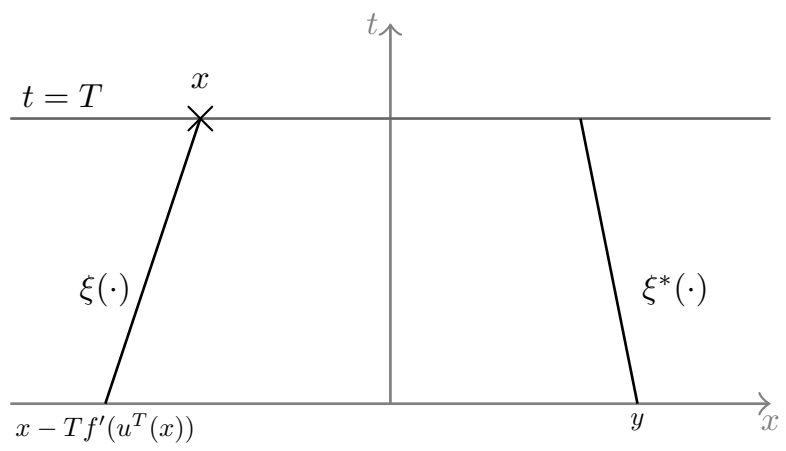

Fig. 4: Plotting of $\xi(\cdot)=\xi_{-}(T-\cdot)$ with $\xi_{-}$the minimal backward generalized characteristic, associated to $u$ (or $u^{T}$ or $u^{*}$ ), emanating from $(T, x)$ and plotting of $\xi^{*}$, the forward generalized characteristic, associated to $u^{*}$, emanating from $(0, y)$ with $(x, y) \in X\left(u^{T}\right) \times \mathbb{R}$ and $x-T f^{\prime}\left(u^{T}(x)\right)<y$.

$\gamma_{1}=\xi$ and $\gamma_{2}=\xi^{*}$,

$$
\begin{aligned}
& \int_{x-T f^{\prime}\left(u^{T}(x)\right)}^{y}\left(u_{0}(s)-u_{0}^{*}(s)\right) d s= \\
& \quad \int_{0}^{T} Q\left(u^{*}\left(s, \xi^{*}(s)+\right), u\left(s, \xi^{*}(s)+\right), u^{*}\left(s, \xi^{*}(s)-\right)\right) d s \\
& \quad+\int_{0}^{T} Q\left(u(s, \xi(s)-), u^{*}(s, \xi(s)-), u(s, \xi(s)-)\right) d s,
\end{aligned}
$$

where $Q$ is defined in (11). From Lemma 2 and (13), for every $(x, y) \in X\left(u^{T}\right) \times \mathbb{R}$ with $x-T f^{\prime}\left(u^{T}(x)\right)<y$,

$$
\int_{x-T f^{\prime}\left(u^{T}(x)\right)}^{y}\left(u_{0}(s)-u_{0}^{*}(s)\right) d s \geq 0 .
$$

Let $(x, y) \in \mathcal{I}\left(u^{T}\right)^{2}$ with $x<y$.

${ }^{1}$ If a rarefaction wave is created at $t=0$, multiple forward generalized characteristics exist.
- There exists a forward generalized characteristic $\xi^{*} \in$ $C^{0,1}([0, T] ; \mathbb{R})$, associated with $u^{*}$, issuing from $(0, x-$ $\left.T f^{\prime}\left(u^{T}(x)\right)\right)$. From [12, Lemma 7.2], $(t, x) \rightarrow$ $S_{t}^{-}\left(u^{T}\right)(x)$ is Lipschitz continuous on any compact $(0, T) \times \mathbb{R}$, thus $\xi^{*}$ is a straight line with slope $f^{\prime}\left(u^{*}\left(\frac{T}{2}, \xi^{*}\left(\frac{T}{2}\right)\right)\right)$. Thus, for every $s \in(0, T)$, $u^{*}\left(s, \xi^{*}(s)-\right)=u^{*}\left(s, \xi^{*}(s)+\right)$.

- From [15, 11.1.3 Theorem], since $u^{T}(y-)=u^{T}(y+)=$ $u(T, y-)=u(T, y+)$ the minimal backward generalized characteristic $\xi_{-}$and the maximal backward generalized characteristic $\xi_{+}$, associated to $u$ (or $u^{T}$ or $u^{*}$ ), emanating from $(T, y)$ coincide over $[0, T]$, i.e $\xi_{-}(t)=\xi_{+}(t)$ for every $t \in[0, T]$. Moreover, $\xi_{-}$is a straight line with slope $f^{\prime}\left(u^{T}(y-)\right)=f^{\prime}\left(u^{T}(y+)\right)$ and $\xi_{-}(T)=$ $y-T f^{\prime}\left(u^{T}(y)\right)$. The function $\xi:[0, T] \rightarrow \mathbb{R}$ defined by $\xi(t)=\xi_{-}(T-t)$ is a forward characteristic associated with $u$, issuing from $\left(0, y-T f^{\prime}\left(u^{T}(y)\right)\right.$.

From [15, Corollary 11.1.2], $\xi$ and $\xi^{*}$ cannot intersect for any $t \in(0, T)$ as seen in Figure 5

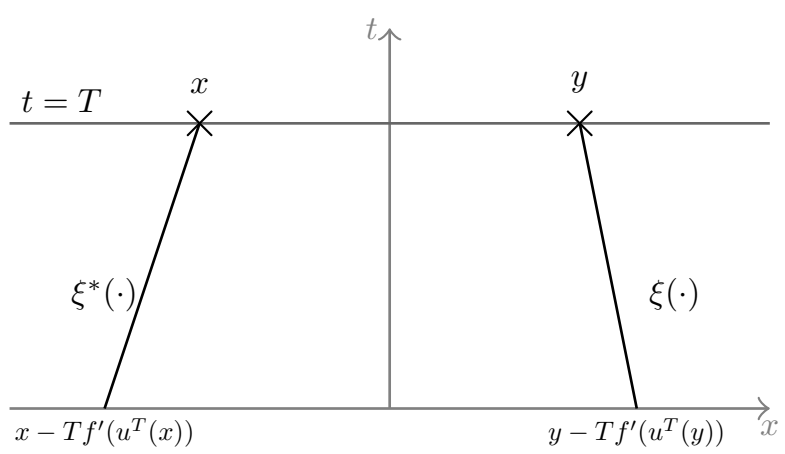

Fig. 5: Plotting of $\xi^{*}$, the forward generalized characteristic, associated to $u^{*}$, emanating from $(0, x)$ and plotting of $\xi(\cdot)=\xi_{+}(T-\cdot)$ with $\xi_{-}$the maximal backward generalized characteristic, associated to $u$ (or $u^{T}$ or $u^{*}$ ), emanating from $(T, y)$ with $(x, y) \in X\left(u^{T}\right)^{2}$ and $x<y$.

Thus, from [15, Theorem 10.2.3] and (12) with $\gamma_{1}=\xi^{*}$ and $\gamma_{2}=\xi$,

$$
\begin{aligned}
& \int_{x-T f^{\prime}\left(u^{T}(y)\right)}^{y-T}\left(u_{0}(s)-u_{0}^{*}(s)\right) d s= \\
& \quad-\int_{0}^{T} Q\left(u(s, \xi(s)+), u^{*}(s, \xi(s)+), u(s, \xi(s)-)\right) d s \\
& \quad-\int_{0}^{T} Q\left(u^{*}\left(s, \xi^{*}(s)-\right), u\left(s, \xi^{*}(s)-\right), u^{*}\left(s, \xi^{*}(s)-\right)\right) d s,
\end{aligned}
$$

From Lemma 2 and (13), for every $(x, y) \in X\left(u^{T}\right)^{2}$ with $x<y$,

$$
\int_{x-T f^{\prime}\left(u^{T}(x)\right)}^{y-T f^{\prime}\left(u^{T}(y)\right)}\left(u_{0}(s)-u_{0}^{*}(s)\right) d s \leq 0 .
$$

From [15, Corollary 11.1.2], we have $x-T f^{\prime}\left(u^{T}(x)\right)<y-$ $T f^{\prime}\left(u^{T}(y)\right)$. Thus, combining (14) with (16), we deduce that, for every $(x, y) \in X\left(u^{T}\right)^{2}$ with $x<y$

$$
\int_{x-T f^{\prime}\left(u^{T}(x)\right)}^{y-T f^{\prime}\left(u^{T}(y)\right)}\left(u_{0}(s)-u_{0}^{*}(s)\right) d s=0 .
$$


The inequality 17) also holds for every $(x, y) \in X\left(u^{T}\right)^{2}$ with $x<y$, whence (9). Assuming that $y<x-T f^{\prime}\left(u^{T}(x)\right)$. There exists a $z \in X\left(u^{T}\right)$ such that $z-T f^{\prime}\left(u^{T}(z)\right)<y$ since a suitable representative of $u^{T} \in L^{\infty}(\mathbb{R})$ satisfies the Oleinik condition (7). Using $z-T f\left(u^{T}(z)\right)<y$ and $z \in X\left(u^{T}\right)$, from 14

$$
\int_{z-T f^{\prime}\left(u^{T}(z)\right)}^{y}\left(u_{0}(s)-u_{0}^{*}(s)\right) d s \geq 0 .
$$

Since $(z, x) \in X\left(u^{T}\right)$, from (8), we have

$$
\int_{z-T f^{\prime}\left(u^{T}(z)\right)}^{x-T f^{\prime}\left(u^{T}(x)\right)}\left(u_{0}(s)-u_{0}^{*}(s)\right) d s=0
$$

Combining (18) and (19), the inequality (14) holds also for $y<x-T f^{\prime}\left(u^{T}(x)\right)$, whence (8).

Since $u^{T} \in L^{\infty}(\mathbb{R})$ satisfies the one-sided Lipschitz condition, from [12, Theorem 3.1], we have $u^{*}(T, \cdot)=u^{T}(\cdot)$. From Lemma 1 applied with $u_{0}$ and $u_{0}^{*}$, we have

$$
\begin{aligned}
& \begin{array}{l}
\int_{\gamma_{1}\left(t_{1}\right)}^{\gamma_{2}\left(t_{1}\right)}\left(u\left(t_{1}, s\right)-u^{*}\left(t_{1}, s\right)\right) d s \\
\quad-\int_{\gamma_{1}\left(t_{2}\right)}^{\gamma_{2}\left(t_{2}\right)}\left(u\left(t_{2}, s\right)-u^{*}\left(t_{2}, s\right)\right) d s= \\
\int_{t_{1}}^{t_{2}} F\left(u\left(t, \gamma_{2}(t)+\right)\right)-F\left(u^{*}\left(t, \gamma_{2}(t)+\right)\right) d t \\
\quad-\int_{t_{1}}^{t_{2}}\left(F\left(u\left(t, \gamma_{1}(t)-\right)\right)-F\left(u^{*}\left(t, \gamma_{1}(t)-\right)\right)\right) d t
\end{array}
\end{aligned}
$$

for every $\left(\gamma_{1}, \gamma_{2}\right) \in C^{0,1}([0, T] ; \mathbb{R})$. Fix $(x, y) \in X\left(u^{T}\right)^{2}$ with $x<y$,

Step 1. Fix $(x, y) \in \mathbb{R} \times X\left(u^{T}\right)$ with $x<y$.

- Let $\xi_{-}$the minimal backward generalized characteristic, associated to $u$, emanating from $(T, x)$. Them, $\xi_{-}$is a straight line with slope $f^{\prime}(u(x-))$ and $\xi_{-}(T)=x-$ $T f^{\prime}(u(x-))$. The function $\xi:[0, T] \rightarrow \mathbb{R}$ defined by $\xi(t)=\xi_{-}(T-t)$ is a forward characteristic associated with $u$, issuing from $\left(0, x-T f^{\prime}(u(x-))\right.$.

- Since $y \in X\left(u^{T}\right)$, we have $u^{T}(x-)=u^{T}(x+)=$ $u^{*}(T, x-)=u^{*}(T, x+)$. Thus, the minimal backward generalized characteristic $\xi_{-}^{*}$ and the maximal backward generalized characteristic $\xi_{+}^{*}$, associated to $u^{*}$, emanating from $(T, y)$ coincide over $[0, T]$, i.e $\xi_{-}^{*}(t)=\xi_{+}^{*}(t)$ for every $t \in[0, T]$. Moreover, $\xi_{+}^{*}$ is a straight line with slope $f^{\prime}\left(u^{T}(x-)\right)=f^{\prime}\left(u^{T}(x+)\right)$ and $\xi_{+}^{*}(T)=$ $y-T f^{\prime}\left(u^{T}(y)\right)$. The function $\xi:[0, T] \rightarrow \mathbb{R}$ defined by $\xi^{*}(t)=\xi_{+}^{*}(T-t)$ is a forward characteristic associated with $u^{*}$, issuing from $\left(0, y-T f^{\prime}\left(u^{T}(y)\right)\right.$.

We now distinguish if $\xi$ doesn't interact with $\xi^{*}$ or not as seen in Figure 6

- Assuming that $\xi$ doesn't interact with $\xi^{*}$ over $(0, T)$. In this case, we have $x-T f^{\prime}(u(x-)) \leq y-T f^{\prime}\left(u^{T}(y)\right)$.

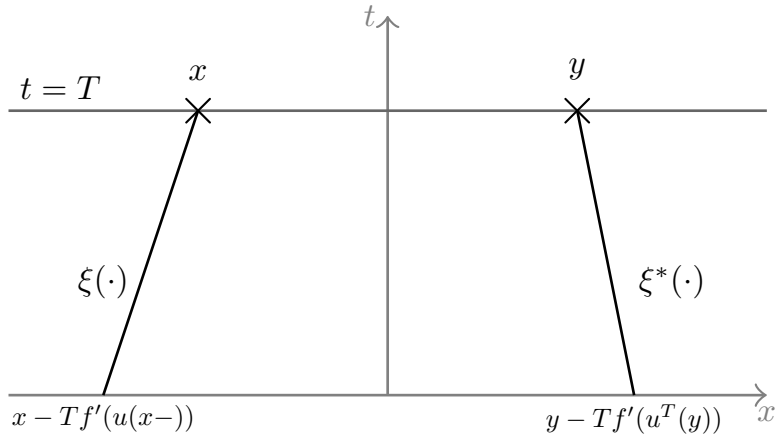

$\xi$ doesn't interact with $\xi^{*}$

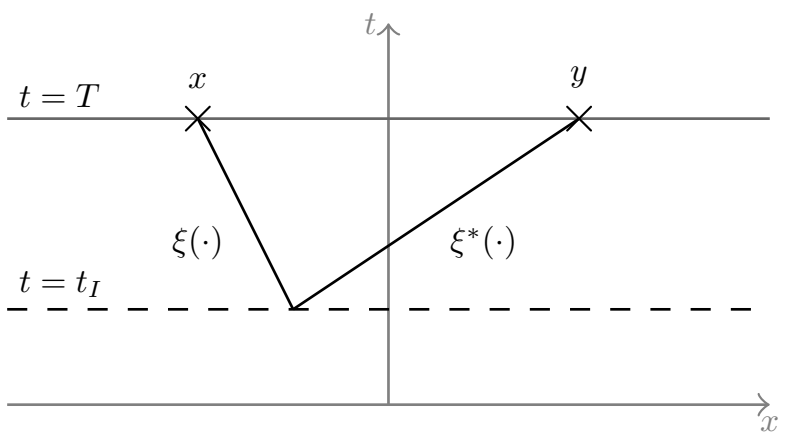

$\xi$ interacts with $\xi^{*}$ at time $t=t_{I}$

Fig. 6: Plotting of $\xi(\cdot)=\xi_{-}(T-\cdot)$ and $\xi^{*}(\cdot)=\xi_{+}^{*}(\cdot)$ where $\xi_{-}$is the minimal backward generalized characteristic, associated to $u$, emanating from $(T, x)$ and $\xi_{+}^{*}(\cdot)$ is the maximal backward generalized characteristic, associated to $u^{*}$, emanating from $(T, y)$ with $(x, y) \in \mathbb{R} \times X\left(u^{T}\right)$ and $x<y$.

From [15, Theorem 10.2.3] and (20) with $t_{1}=0, t_{2}=T$, $\gamma_{1}=\xi$ and $\gamma_{2}=\xi^{*}$,

$$
\begin{aligned}
& \int_{x}^{y}\left(u^{*}(T, s)-u(T, s)\right) d s \\
& \quad+\int_{x-T f^{\prime}(u(x-))}^{y-T f^{\prime}\left(u^{T}(y)\right)}\left(u_{0}(s)-u_{0}^{*}(s)\right) d s= \\
& \int_{0}^{T} Q\left(u^{*}\left(s, \xi^{*}(s)+\right), u\left(s, \xi^{*}(s)+\right), u^{*}\left(s, \xi^{*}(s)-\right)\right) d s \\
& \quad+\int_{0}^{T} Q\left(u(s, \xi(s)-), u^{*}(s, \xi(s)-), u(s, \xi(s)-)\right) d s,
\end{aligned}
$$

where $Q$ is defined in (11). Applying (8) with $x=y$ and $y=x-T f^{\prime}(u(x-))$, we have

$$
\int_{x-T f^{\prime}(u(x-))}^{y-T f^{\prime}\left(u^{T}(y)\right)}\left(u_{0}(s)-u_{0}^{*}(s)\right) d s \leq 0 .
$$

From Lemma 2, (21) and 22), $(x, y) \in \mathbb{R} \times X\left(u^{T}\right)$ with $x<y$,

$$
\int_{x}^{y}\left(u^{*}(T, s)-u(T, s)\right) d s \geq 0
$$

- Assuming that $\xi$ interacts with $\xi^{*}$ at time $t_{I} \in(0, T)$. For every $t \in\left(t_{I}, T\right)$, we have $\xi(t) \leq \xi^{*}(t)$. From [15 Theorem 10.2.3] and 20] with $t_{1}=t_{I}, t_{2}=T, \gamma_{1}=\xi$ 
and $\gamma_{2}=\xi^{*}$

$$
\begin{aligned}
& \int_{x}^{y}\left(u^{*}(T, s)-u(T, s)\right) d s \\
& =\int_{t_{I}}^{T} Q\left(u^{*}\left(s, \xi^{*}(s)+\right), u\left(s, \xi^{*}(s)+\right), u^{*}\left(s, \xi^{*}(s)-\right)\right) d s \\
& \quad+\int_{t_{I}}^{T} Q\left(u(s, \xi(s)-), u^{*}(s, \xi(s)-), u(s, \xi(s)-)\right) d s .
\end{aligned}
$$

From Lemma 2, (24), for every $(x, y) \in \mathbb{R} \times X\left(u^{T}\right)$ with $x<y$,

$$
\int_{x}^{y}\left(u^{*}(T, s)-u(T, s)\right) d s \geq 0
$$

From (23) and 25], we conclude that for every $(x, y) \in \mathbb{R} \times$ $X\left(u^{T}\right)$ with $x<y$,

$$
\int_{x}^{y}\left(u^{*}(T, s)-u(T, s)\right) d s \geq 0
$$

Step 2. Fix $(x, y) \in X\left(u^{T}\right)^{2}$ with $x<y$.

- Since $x \in X\left(u^{T}\right)$, we have $u^{T}(x-)=u^{T}(x+)=$ $u^{*}(T, x-)=u^{*}(T, x+)$. Thus, the minimal backward generalized characteristic $\xi_{-}^{*}$ and the maximal backward generalized characteristic $\xi_{+}^{*}$, associated to $u^{*}$, emanating from $(T, x)$ coincide over $[0, T]$, i.e $\xi_{-}^{*}(t)=\xi_{+}^{*}(t)$ for every $t \in[0, T]$. Moreover, $\xi_{-}^{*}$ is a straight line with slope $f^{\prime}\left(u^{T}(x-)\right)=f^{\prime}\left(u^{T}(x+)\right)$ and $\xi_{-}^{*}(T)=$ $x-T f^{\prime}\left(u^{T}(x)\right)$. The function $\xi^{*}:[0, T] \rightarrow \mathbb{R}$ defined by $\xi^{*}(t)=\xi_{-}^{*}(T-t)$ is a forward characteristic associated with $u^{*}$, issuing from $\left(0, x-T f^{\prime}\left(u^{T}(x)\right)\right.$.

- Let $\xi_{+}$the maximal backward generalized characteristic, associated to $u$, emanating from $(T, y)$. Them, $\xi_{+}$is a straight line with slope $f^{\prime}(u(y+))$ and $\xi_{+}(T)=y-$ $T f^{\prime}(u(y+))$. The function $\xi:[0, T] \rightarrow \mathbb{R}$ defined by $\xi(t)=\xi_{+}(T-t)$ is a forward characteristic associated with $u$, issuing from $\left(0, y-T f^{\prime}(u(y+))\right.$.

We now distinguish if $\xi$ doesn't interact with $\xi^{*}$ or not as seen in Figure 7

- Assuming that $\xi^{*}$ doesn't interacts with $\xi$ over $(0, T)$. In this case, we have $x-T f^{\prime}\left(u^{T}(x)\right) \leq y-T f^{\prime}(u(y)+)$. From [15, Theorem 10.2.3] and (20) with $t_{1}=0, t_{2}=T$, $\gamma_{1}=\xi^{*}$ and $\gamma_{2}=\xi$,

$$
\begin{aligned}
& \int_{x}^{y}\left(u^{*}(T, s)-u(T, s)\right) d s \\
& \quad+\int_{x-T f^{\prime}(u(y+))}^{\left.y-T f^{\prime}(x)\right)}\left(u_{0}(s)-u_{0}^{*}(s)\right) d s= \\
& -\int_{0}^{T} Q\left(u(s, \xi(s)+), u^{*}(s, \xi(s)+), u(s, \xi(s)-)\right) d s \\
& -\int_{0}^{T} Q\left(u^{*}\left(s, \xi^{*}(s)-\right), u\left(s, \xi^{*}(s)-\right), u^{*}\left(s, \xi^{*}(s)-\right)\right) d s
\end{aligned}
$$

where $Q$ is defined in (11). Applying (8) with $x=x$ and $y=y-T f^{\prime}(u(y+))$, we have

$$
\int_{x-T f^{\prime}\left(u^{T}(x)\right)}^{y-T f^{\prime}(u(y+))}\left(u_{0}(s)-u_{0}^{*}(s)\right) d s \geq 0 .
$$

From Lemma 27 and 28, for every $(x, y) \in X\left(u^{T}\right)^{2}$ with $x<y$,

$$
\int_{x}^{y}\left(u^{*}(T, s)-u(T, s)\right) d s \leq 0
$$
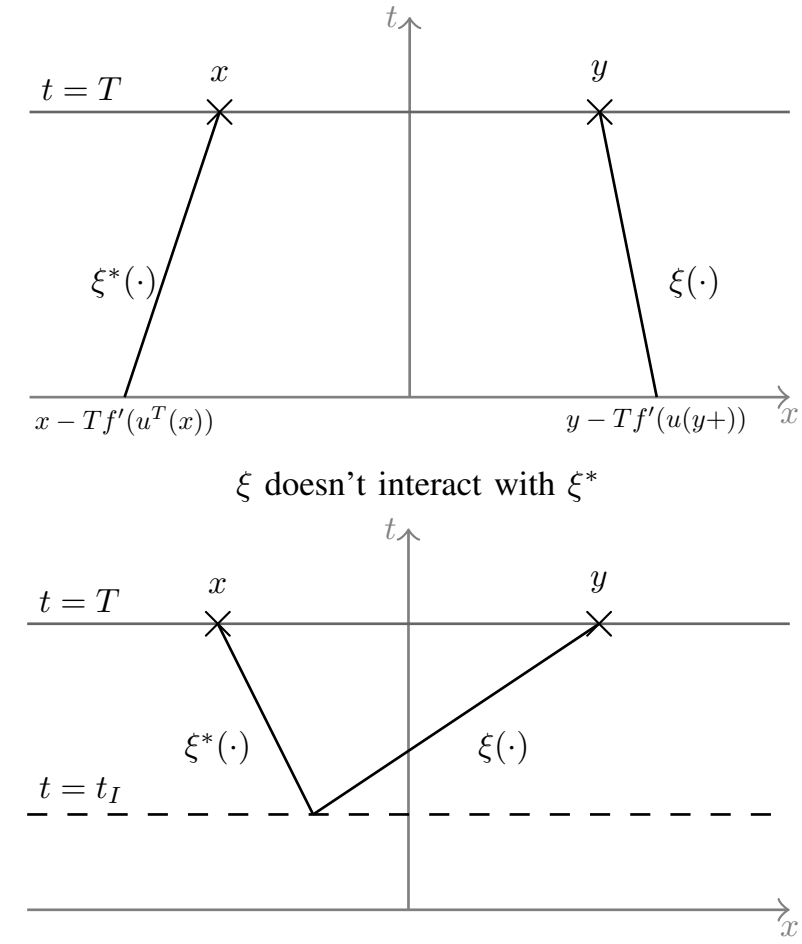

$\xi$ interacts with $\xi^{*}$ at time $t=t_{I}$

Fig. 7: Plotting of $\xi^{*}=\xi_{-}^{*}(\cdot)$ and $\xi=\xi_{+}(T-\cdot)$ where $\xi_{-}$is the minimal backward generalized characteristic, associated to $u^{*}$, emanating from $(T, x)$ and $\xi_{+}(\cdot)$ is the maximal backward generalized characteristic, associated to $u$, emanating from $(T, y)$ with $(x, y) \in X\left(u^{T}\right)^{2}$ and $x<y$.

- Assuming that $\xi^{*}$ interacts with $\xi$ at time $t_{I} \in(0, T)$. For every $t \in\left(t_{I}, T\right)$, we have $\xi^{*}(t) \leq \xi(t)$. From [15 Theorem 10.2.3] and 20] with $t_{1}=t_{I}, t_{2}=T, \gamma_{1}=\xi^{*}$ and $\gamma_{2}=\xi$,

$$
\begin{aligned}
& \int_{x}^{y}\left(u^{*}(T, s)-u(T, s)\right) d s= \\
& -\int_{t_{I}}^{T} Q\left(u(s, \xi(s)+), u^{*}(s, \xi(s)+), u(s, \xi(s)-)\right) d s \\
& -\int_{t_{I}}^{T} Q\left(u^{*}\left(s, \xi^{*}(s)-\right), u\left(s, \xi^{*}(s)-\right), u^{*}\left(s, \xi^{*}(s)-\right)\right) d s,
\end{aligned}
$$

From Lemma 2. (30), for every $(x, y) \in X\left(u^{T}\right)^{2}$ with $x<y$,

$$
\int_{x}^{y}\left(u^{*}(T, s)-u(T, s)\right) d s \leq 0
$$

From 29] and (31), we conclude that for every $(x, y) \in$ $X\left(u^{T}\right)^{2}$ with $x<y$,

$$
\int_{x}^{y}\left(u^{*}(T, s)-u(T, s)\right) d s \leq 0
$$

Combining 26 with 32, we deduce that, for every $(x, y) \in X\left(u^{T}\right)$ with $x<y$,

$$
\int_{x}^{y}\left(u^{*}(T, s)-u(T, s)\right) d s=0 .
$$

Step 3. From [15, Theorem 11.2.2], $u(T, \cdot) \in B V_{\text {loc }}(\mathbb{R})$ and $u^{*}(T, \cdot) \in B V_{\text {loc }}(\mathbb{R})$. Thus, $X\left(u^{T}\right)$ has Lebesgue measure 
0 . The equality (33) holds for a.e $(x, y) \in \mathbb{R}$ with $x<y$. Since $y \rightarrow \int_{x}^{y} u(T, s) d s$ and $y \rightarrow \int_{x}^{y} u^{*}(T, s) d s$ are absolute continuous functions, (33) holds for every $y \in \mathbb{R}$. Using the fundamental theorem of calculus, we deduce that, for a.e $y \in$ $\mathbb{R}$,

$$
\begin{gathered}
u(T, y)=u^{*}(T, y) . \\
\text { V. CONCLUSION }
\end{gathered}
$$

\section{CONCLUSION}

In this paper, we construct theoretically and numerically the set of initial data yielding solutions of Burgers equation (2) that coincide with a given target. When the augmented Burgers equation (1) is reduced to Burgers equation (2), this allows to find the set of near-pressure disturbances $P_{0}$ leading to the bound signature $P^{T}$ optimized for minimum pressure rise. The proof is based on a backward-forward method and generalized backward characteristics.

Let us address some related open questions and possible extensions of this work.

In the development of low-boom supersonic airplane, it would be interesting to understand the impact of the nonlocal term and the zero-order term in (1) for the initial data identification problem. Moreover, some constraints on the admissible set of initial data could be imposed to ensure feasible aircraft design.

Since we don't need Lax-Hopf formula as in [12, Theorem 4.1], we may extend our approach to systems of conservation laws in one dimension (Euler equations, SaintVenant equations, Aw-Rascle-Zhang model). Note that, as soon as the backward-forward operator $S_{T}^{+}\left(S_{T}^{-}\right)$is welldefined, $S_{T}^{+}\left(S_{T}^{-}\right)\left(u^{T}\right)$ may give a good candidate to solve the initial data identification of systems of conservation laws.

\section{REFERENCES}

[1] Navid Allahverdi, Alejandro Pozo, and Enrique Zuazua. Numerical aspects of large-time optimal control of burgers equation. ESAIM: Mathematical Modelling and Numerical Analysis, 50(5):1371-1401, 2016.

[2] Navid Allahverdi, Alejandro Pozo, and Enrique Zuazua. Numerical aspects of sonic-boom minimization. A Panorama of Mathematics: Pure and Applied, 658:267, 2016.

[3] Juan J Alonso and Michael R Colonno. Multidisciplinary optimization with applications to sonic-boom minimization. Annual Review of Fluid Mechanics, 44:505-526, 2012.

[4] François Bouchut and François James. One-dimensional transport equations with discontinuous coefficients. Nonlinear Analysis, 32(7):891, 1998.

[5] François Bouchut and François James. Differentiability with respect to initial data for a scalar conservation law. In Hyperbolic problems: theory, numerics, applications, pages 113-118. Springer, 1999.

[6] Alberto Bressan and Rinaldo M Colombo. Decay of positive waves in nonlinear systems of conservation laws. Annali della Scuola Normale Superiore di Pisa-Classe di Scienze, 26(1):133-160, 1998.

[7] Alberto Bressan and Andrea Marson. A maximum principle for optimally controlled systems of conservation laws. Rendiconti del Seminario Matematico della Universita di Padova, 94:79-94, 1995.

[8] Alberto Bressan and Andrea Marson. A variational calculus for discontinuous solutions of systems of conservation laws. Communications in partial differential equations, 20(9):1491-1552, 1995.

[9] Carlos Castro, Francisco Palacios, and Enrique Zuazua. An alternating descent method for the optimal control of the inviscid burgers equation in the presence of shocks. Mathematical Models and Methods in Applied Sciences, 18(03):369-416, 2008.
[10] Carlos Castro, Francisco Palacios, and Enrique Zuazua. Optimal contro and vanishing viscosity for the burgers equation. In Integral Methods in Science and Engineering, Volume 2, pages 65-90. Springer, 2010.

[11] Robin Olav Cleveland. Propagation of sonic booms through a real stratified atmosphere. PhD thesis, Citeseer, 1995.

[12] Rinaldo Colombo and Vincent Perrollaz. Initial data identification in conservation laws and hamilton-jacobi equations. arXiv preprint arXiv:1903.06448, 2019.

[13] C. Dafermos. Generalized characteristics and the structure of solutions of hyperbolic conservation laws. Indiana Univ. Math. J., 26:1097-1119, 1977.

[14] Constantine M Dafermos. Polygonal approximations of solutions of the initial value problem for a conservation law. Journal of Mathematical Analysis and Applications, 38(1):33-41, 1972.

[15] Constantine M Dafermos. Hyperbolic conservation laws in continuum physics, volume 325 of grundlehren der mathematischen wissenschaften [fundamental principles of mathematical sciences], 2010.

[16] Carlos Esteve and Enrique Zuazua. The inverse problem for hamiltonjacobi equations and semiconcave envelopes. SIAM Journal on Mathematical Analysis, 52(6):5627-5657, 2020.

[17] Shyam Sundar Ghoshal, GD Veerappa Gowda, et al. Exact controllability of scalar conservation laws with strict convex flux. Mathematical Control \&amp; Related Fields, 4(4):401, 2014

[18] Olivier Glass. An extension of oleinik's inequality for general 1d scalar conservation laws. Journal of Hyperbolic Differential Equations, 5(1):113, 2008.

[19] Laurent Gosse and Enrique Zuazua. Filtered gradient algorithms for inverse design problems of one-dimensional burgers equation. In Innovative algorithms and analysis, pages 197-227. Springer, 2017.

[20] Stanislav N Kružkov. First order quasilinear equations in several independent variables. Mathematics of the USSR-Sbornik, 10(2):217, 1970.

[21] Peter D Lax. Hyperbolic systems of conservation laws ii. Communications on pure and applied mathematics, 10(4):537-566, 1957.

[22] Andrea Minelli, Itham Salah el Din, and Gerald Carrier. Advanced optimization approach for supersonic low-boom design. In 18th AIAA/CEAS Aeroacoustics Conference (33rd AIAA Aeroacoustics Conference), page 2168, 2012.

[23] Olga Arsen'evna Oleinik. Discontinuous solutions of non-linear differential equations. Uspekhi Matematicheskikh Nauk, 12(3):3-73, 1957.

[24] Joseph Pawlowski, David Graham, Charles Boccadoro, Peter Coen, and Domenic Maglieri. Origins and overview of the shaped sonic boom demonstration program. In 43rd AIAA Aerospace Sciences Meeting and Exhibit, page 5, 2005.

[25] Sriram Rallabhandi. Sonic boom adjoint methodology and its applications. In 29th AIAA Applied Aerodynamics Conference, page 3497, 2011.

[26] Sriram K Rallabhandi. Advanced sonic boom prediction using the augmented burgers equation. Journal of Aircraft, 48(4):1245-1253, 2011.

[27] Gerald Beresford Whitham. The flow pattern of a supersonic projectile. Communications on pure and applied mathematics, 5(3):301-348, 1952 